\title{
Bacterial cell wall nature and its mode of resistance against antibiotic drugs: An Overview
}

\author{
Saif Ali ${ }^{1}$ Bhavtosh Sharma ${ }^{2 *}$ Anita Rawat ${ }^{2}$ \\ ${ }^{1}$ Shri Guru Ram Rai University (SGRRU), Dehradun, Uttarakhand, India \\ ${ }^{2}$ Uttarakhand Science Education and Research Centre (USERC), Dehradun, Uttarakhand, India \\ *Corresponding author: bhavtoshchem@gmail.com
}

Received: 1.12.2021; Revised: 16.12.2021; Accepted:26.12.2021

(C)Society for Himalayan Action Research and Development

\begin{abstract}
Bacterial cell wall is made up of two derivatives of NAG (N-acetylglucosamine) and NAM (Nacetylmuramic) which forms peptidoglycan. On the basis of peptidoglycan bacteria are classified into two main categories: Gram-positive and Gram-negative. But there are some other bacteria which are devoid of cell wall like PPLOs (pleuropneumonia like organism) and some are L-form which do have a cell wall but they can switch to cell wall deficient state and vice-versa. L- form bacteria can change shapes to avoid antibiotics. Bacterial strain ST144 and ST782 were tested on media with or without osmo-protection in the presence and absence of Fosfomycin and showed that bacteria can switched to L-form and revert back to walled state after antibiotic treatment and causing recurrent UTI (Urinary tract infection) in patients. Inappropriate use of antibiotics and immunity effectors favour bacterial L- form. Bacteria have different mechanism of avoiding antibiotics but changing the morphology to L-form make difficult for antibiotics to identify the cell wall which makes bacteria to persist more in human body to causing full blown infection. Mutation caused by disassembly of ribosomal sub-unit can also helps the bacteria to survive in multi-drug environment. The current article highlights the nature of bacterial cell wall and its nature against antibiotic drugs in human beings.
\end{abstract}

Keywords: L-form bacteria, UTI, Peptidoglycan, Antibiotics, Ribosomal mutation, Cell wall deficient bacteria, Antibiotic drugs

\section{Introduction}

The bacterial cell wall plays an important role providing the shape and rigidity to bacteria. The cell wall is made up of peptidoglycan which is a defining feature of bacteria. Peptidoglycan is a complex structure made up of polymeric carbohydrates and amino acids. The two derivatives of glucose NAG and NAM with a pentapeptide coming off NAM constitute peptidoglycan. A molecule named bactoprenol transports the NAG and NAM strands (synthesized in cytosol) across the cytoplasmic membrane where it is arranged by glycosyltransferase (GTase) and transpeptidase (TPase) enzymes (Scheffers and Pinho,2005). On the basis of peptidoglycan, different bacteria are classified into Gram positive and Gram- negative bacteria type. Gram positive bacteria stains crystal violet while Gram negative bacteria stains safranin. In Gram negative bacteria, peptidoglycan is thin (7-8nm) surrounded by two plasma membrane and a special LPS molecule on the outer surface of plasma membrane (Purcell,2016). LPS (or endotoxin) is only found in Gram negative bacteria which are toxic to animals. Whereas in Gram positive bacteria $(20-80 \mathrm{~nm}$ thick) peptidoglycan is composed of several layers of peptidoglycan (Purcell,2016). Vertically through the peptidoglycan, a group of molecules called teichoic acid is embedded in it which is unique to Gram positive. Teichoic acids are linear polymers of polyglycerol or polyribitol 
substituted with phosphates and few amino acids as well as sugars. It gives negative charge to overall gram-positive cell which is due to presence of phosphodiester bond between its monomers. These cell wall gives the ligands for attachment and receptor sites for viruses and antibiotics. That's important because many antibiotics kills bacteria by weakening their cell wall like penicillin, so they are quite resistant to broad spectrum antibiotics.

PPLOs (now called mycoplasmas) which are quite spherical in shape and are devoid of cell wall. They are consequently placed in a separate class Mollicutes. Mycoplasmas are the smallest self-replicating organism with smallest genomes (about 500 to 1000 genes), they are low in guanine and cytosine. Mycoplasmas possess surface antigens such as lipoproteins, membrane proteins, glycolipids and lipoglycan which are exposed on cell surface and are the major antigenic determinants in mycoplasmas. Antisera containing antibodies to these components reduce the growth as well as the metabolism of the mycoplasmas and in presence of complement, cause the lysis of the organism (Razin,1996). But if we talk about L- form bacteria which is also CWD (cell wall deficient bacteria). These bacteria were first discovered by Emmy Klieneberger in 1935 (Klineberger,1935). She named the bacteria Lform or L-shaped in honour of Listeria Institute in London where she was working. She found the bacteria isolated from rat's blood called Streptobacillus moniliformis along with some pleomorphic organism. Her colleague, Louis Dienes, revealed that pleomorphic variants had actually arise from bacilli and the bacteria has the ability to switch between the two morphological forms (Dienes, 1939).

L- form bacteria are different from mycoplasmas because they are not derived from bacteria that normally have cell wall. Lform bacteria are divided into two forms - one is unstable L- form which is capable of dividing and can revert back the original walled morphology and other one is stable Lform which are unable to revert to original morphology. Most of the bacteria divide by binary fission. This process requires a cell wall and Fts $\mathrm{Z}$ gene. But L- form bacteria grow and divide without both these structures. L- forms are pleomorphic and osmotically sensitive. However, they are completely resistant to wide range of antibiotics that work on cell wall (Gilpin et al.,1973).

Many antibiotics affect the bacteria by weakening their cell wall. Bacteria hide themselves by changing the forms in human body to avoid antibiotics. So, it quite difficult to identify the L- form bacteria. L- form bacteria are osmo-sensitive so they require osmo-protective media for their growth. Research showed that L- form bacteria can revert back to walled state within five hours (Leaver et al.,2012; Clasener,1972; Onwuamaegbu et al.,2005;Allan et al.,2009; 
Domingue,2010; Wu et al.,2016). According to World Health Organization, the resistance nature in bacteria is identified as the major menaces to public health (WHO, 2015).

Current study showed that 29 out of 30 patients are associated with UTIs (Urinary Tract Infection) of different L- forms bacterial species including Escherichia coli, Enterococcus, Staphylococcus and Enterobacter. L- form bacteria are fragile and weak but some are sensitive which hide inside the body. Isolated L- form bacteria from UTI patient reform their cell wall within five hours, after the effect of antibiotic is decreased. In 2018, a publication by Errington team showed that our immune system induces L-form switching to some extent. Dr. Mickiewicz explained that a normal healthy person can completely eradicate the L- form bacteria by their immune system. But in elderly patient, their immune system is weaker, so there is a chance of surviving the $\mathrm{L}$ - form bacteria inside the host and cause recurring UTI (Mickiewicz et al.,2018; Wu et al.,2016).

\section{Mechanism of $L$ - form switching}

L- form switching would only be possible if Lforms survive in urine or tissues. The osmolality of urine of a healthy person oscillates between about 400 and $800 \mathrm{mOsm} / \mathrm{kg}$ while L-form bacteria requires osmo-protective environment (which is about 500mOsm/1) (Plischke et al.,2014; Losos et al.,2008). A research was conducted to check whether patients have the potential to survive the L- form bacteria in urine. ST144 bacteria was taken on agarose pad made with filtered urine of a healthy person with added Fosfomycin and observed by time-lapse microscopy. The bacteria were able to switch to $\mathrm{L}$ - form state and proliferate but less efficiently than optimized L- form growth media. ST144 L- form cells viability and their regeneration to the walled state had been checked by placing the cells in liquid filtered urine added with Fosfomycin and incubated overnight. Next day, it was confirmed by phase contrast microscopy, showing the presence of intact L- form cells. The L- form cells were plated on media with and without osmo-protection and incubated for 24 hours at $37^{\circ} \mathrm{C}$. Small colonies can be seen only on osmo-protective media. Microscopic examination showed that mostly walled bacteria, some L -form and intermediate types were seen (Rojas et al.,2018). The L -form bacteria could now grow well on non-osmoprotective medium which proved that they had revert back to walled state. These results give a base to the research that isolated bacteria from recurring UTI patients can survive in urine in the presence of Fosfomycin and can revert back to walled state after the effect of antibiotic is diminished(Fig1) (Domingue and Woody,1997; Kawai et al.,2014; Mercier et al.,2014).

The growth of L-form bacteria is quite slower than walled state bacteria and needs osmoprotective media for growth. Many varieties of bacteria including Escherichia coli can alter their cell wall to L-form (Wu et al.,2016). 
Immune-effectors like Lysozyme and some specific antibiotics which inhibit cell wall synthesis in bacteria such as Fosfomycin and D-cycloserine can also induces L-form switch (Typas et al.,2011).

A test was conducted to determine whether Lform bacteria are capable of switching to walled state or not. The isolated E. coliST782 is grown on media with or without osmoprotection in the presence of Fosfomycin. The time-lapse imaging shows, in absence of osmo-protection, cells started bulging and undergone several divisions and after $2.5 \mathrm{hr}$, all cells became lysed. On the other hand, cells on osmo-protective media undergo series of divisions and continued to grow in the absence of cell wall. The transition to L-form from walled state was completed after $3 \mathrm{hr}$ of incubation.

The L-form bacteria were capable of switching to walled state in recurrent infection after the antibiotic treatment (Fig 1). In the absence of Fosfomycin, L-forms were grown on osmoprotective media. Time-lapse imaging shows that cells undergo several rounds of irregular division for 40 minutes and after that cells extended to their normal diameter and cell wall began to reappear. The elongated cells continued to divide normally by binary fission (Drage et al.,2019).

\section{Resistance nature against Antibiotics}

Bacteria are the oldest living organism on this planet. The smallest thing we still consider life, they are the masters of survival and are ubiquitous. Bacteria inhabits the human body and help us in many ways to survive, but some are parasitic, spread quickly and causes different diseases which are fatal to us. Millions of people die as a result of bacterial infections. Together with vaccinations, antibiotics revolutionized medicine and saved millions of lives. Antibiotics kill the vast majority of susceptible bacteria fairly quickly,leaving only small group of survivors that our immune system then deals with easily. Antibiotics disrupts the complex machinery of bacteria by interfering their metabolism, slowing down their growth significantly, so they are less of a threat. Some antibiotics attack DNA and prevent it from being replicated, which stops bacterial multiplication, ultimately killing them. Or by simply ripping the outer layer of the bacteria to shreds so that their inside spills out and they die quickly. But now, evolution is making things more complicated. By pure random chance, a small minority of the bacteria invading our body might have evolved a way to protect themselves.Bacteria immune to antibiotics might spread their immunity if they escape our body immune system. Bacteria immune to multiple antibiotics are superbugs, a variety of superbugs already exist in the world (Harms et al.,2016). Bacteria is genetically flexible which allows them to respond to wide range of environmental stress including antibiotics which risks their existence. Bacteria share the ecological niche with antimicrobial producing bacteria which 


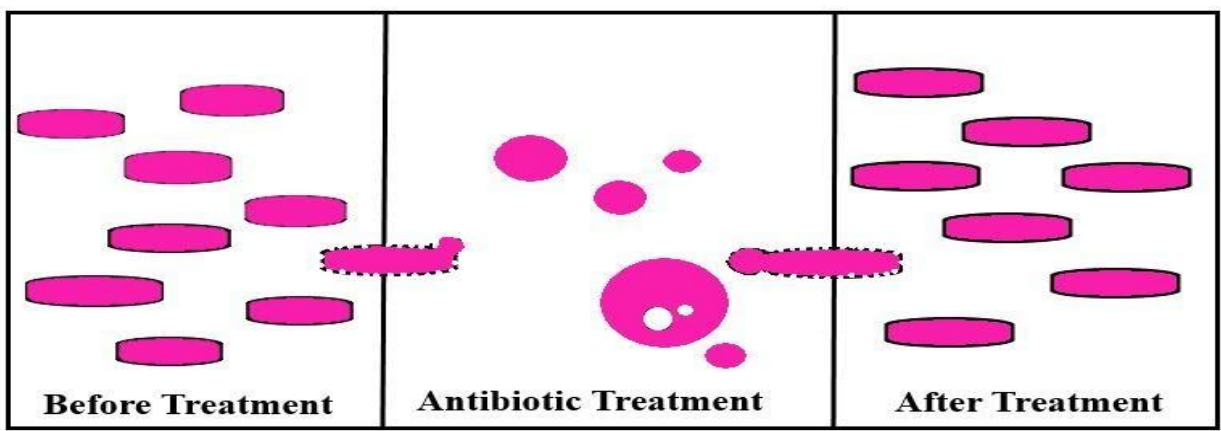

Fig 1. Diagram showing mechanism of L- form switching for recurrence of bacterial infections. UTI causing bacteria are treated with cell wall targeting antibiotics which causes degradation of cell wall and development of L-forms. After antibiotic treatment bacteria can regenerate their cell wall and causes recurrent UTI. The bacterial cell wall is indicated by black lines.

\section{Mutant Ribosomes and the action of antibiotic drug}

The diversity of effects produces by the aminoglycoside and the ingenuity with which they have been pursued have made the streptomycin story the most fascinating and complicated one in the field of anti-ribosomal action. When streptomycin drug is given to bacteria, we kill the bacteria, if bacteria somehow the DNA while they were replicating made a mistake and gained a mutation become resistant to this drug. But there is also an extreme case of resistance where bacteria is resistant and we take the drug out and bacteria dies without knowing it became dependent on drug. Ribosome had a "deocoding site" that sensed codon-anticodon base pairs. Streptomycin is a bactericidal drug and distorts the decoding center of ribosome. Ribosomal sub-units confers resistance to several diverse antibiotic. The changes in the ribosome does not mistranslates but creates misassembly of the ribosomes in which leads to dramatic shift in the transcriptional program and imparts the resistance to different antibiotic with different mode of action. Even the presence of small population of mutant ribosome could allow for the survival of large population. (Gomez et al.,2017)

\section{Results and Discussion}

Gram- negative bacteria have already evolutionary kind of resistance against penicillin antibiotic. Penicillin binds to the proteins within the peptidoglycan layer, that is why penicillin is more effective against gram positive bacteria. However, the penicillin is not effective against gram negative bacteria because of thin peptidoglycan cell wall and extra lipid membrane. Bacteria not only possess the chromosomes but also have a special extra circular DNA called as plasmid and these plasmids are incorporated with resistance genes. Bacteria became resistant to antibiotics by incorporating their extra circular DNA to main chromosomal DNA. To become 
resistant bacterial plasmid generally incorporated into main DNA. Plasmids synthesizes mRNA having information for the resistance towards the antibiotic drugs which is further read by ribosomes to translate the polypeptide of particular proteins. Finally, they read by ribosomes which make polypeptides or protein structures. These protein structures have particular mechanism for targeted antibiotic. The translated polypeptide can form into antibiotic degrading enzymes like beta-lactamase, penicillin have beta- lactam rings and encounters beta lactamase in bacteria. The beta-lactamase breaks off the beta-lactam rings by causing the penicillin to become inactive. There are many types of beta-lactamases found in bacteria like Escherichia coli. Bacteria may also form protein structures called efflux pump. Some have efflux pump on their membrane. Antibiotic drug tetracycline inhibits the protein synthesis, so bacteria pumped out tetracycline using efflux pump.

Some bacteria have TPases enzyme embedded in peptidoglycan layer which are penicillin binding proteins (PBP). These proteins are targeted by beta lactams antibiotics (Lovering et al.,2012). Penicillin contain beta-lactam ring and bacteria have penicillin binding protein which basically change the structures of proteins, penicillin is unable to bind the protein, because protein structure is change. In MRSA (Methicillin resistant staphylococcus aureus), bacteria which is resistant to antibiotic methicillin. Methicillin does not work because the protein on which methicillin binds is actually modified by bacteria (Reygaert,2018). Other way is Horizontal gene transfer which includes Conjugation, Transduction and Transformation. Conjugation involves the transfer of resistant genes by replication of plasmid which is passed on to other bacteria. Bacteriophage affects bacteria and release their genetic material inside them and replicate inside the bacteria, while bacteria contains resistant genes, these resistant genes accidently pack inside the viral bodies. When such virus infects other bacteria, the resistant genes get transferred into it and make it resistant to antibiotics. This mechanism is called transduction which may be generalized or specialized, depending upon viruses. Bacteria containing resistant genes, when they die, their genetic material can be easily picked up by other bacteria through Transformation which again make the other bacteria resistant to antibiotics.

Many drugs like penicillin, cephalosporins and Vancomycin are bactericidal in nature means they kill the cell by inhibiting the cell wall synthesis. All these drugs have different mechanism of inhibiting cell wall synthesis but one good thing about these drugs are - they won't affect human cells because human cells does not have peptidoglycan or cell wall. Antibiotic that disrupts cell wall are pretty extensive and resulting in cell death. Rifamycin prevents RNA synthesis in bacteria so bacteria cannot do transcription and translation. 
Bacteria used to make protein in order to survive, however there are antibiotics that target either the 30s subunit or 50s subunit of ribosomes. Erythromycin and chloramphenicol targets 50s subunit whereas tetracycline, streptomycin and gentamycin targets 30s subunit of ribosomes, these are bacteriostatic antibiotics. So, bacteria cannot make proteins which make bacteria alive but it won't be able to make anything.

\section{Conclusion}

Humans are evolved so far but bacteria already evolved billions of years ago, they now just modifying their mechanism to survive against antibiotics. Recurrent UTI is a serious problem for global health and bacteria which causes UTI have become resistant to many antibiotics. And now the major problem is switching of bacteria to L- form to avoid targeting by antibiotics is a serious concern because many antibiotics kill bacteria by inhibiting their cell wall synthesis like penicillin, cephalosporins etc. If we use bacteriostatic drugs against these L- form bacteria it might be helpful to some extent but these drugs can interfere with human DNA and RNA synthesis and can cause less common side effects like abnormal blood clotting if cephalosporins were taken, sensitivity to sunlight when tetracycline is taken. The above results showed that in presence of Fosfomycin bacteria stays in Lform and when the effect of antibiotic diminished, bacteria again revert back to walled state causing long term infection in patients. So, we have to take step forward to minimize the use of antibiotics or we have to find the alternate of antibiotics. Carbapenems can be given to the patients only in severe infections as they are last line antibiotic agents and have greatest potency against bacteria. Antibiotics having target action on ribosomes can confer resistance to bacteria from multiple drugs as small mutation in ribosomal sub-unit can transfer the resistance to large population for their survival.

\section{Conflict of Interest}

The authors declare that they have no conflict of interest.

\section{References}

Allan, E. J., Hoischen, C. \& Gumpert, J. (2009)Bacterial L-forms. Adv. Appl. Microbiol. 68, 1-39 .

Clasener, H.(1972) Pathogenicity of the Lphase of bacteria. Annu. Rev. Microbiol.26, 55-84 .

Dienes L. 1939 L Organisms of Klieneberger and Streptobacillus moniliformis. J. Infect. Dis. 65,24-42.

Domingue, Sr, G.J. and Woody, H.B. (1997) Bacterial persistence and expression of disease. Clin. Microbiol. Rev. 10, 320344.

Domingue, G. J. (2010).Demystifying pleomorphic forms in persistence and expression of disease: Are they bacteria, and is peptidoglycan the solution? Discov. Med. 10, 234-246. 
Domínguez-Cuevas, P., Mercier, R., Leaver, M., Kawai, Y. and Errington, J. (2012) The rod to L-form transition of Bacillus subtilis is limited by a requirement for the protoplast to escape from the cell wall sacculus. Mol. Microbiol. 83, 5266.

Domingue, G. J. Sr. \& Woody, H. B.(1997) Bacterial persistence and expression of disease. Clin. Microbiol. Rev. 10, 320344 .

Errington, J., Mickiewicz, K., Kawai, Y. \& $\mathrm{Wu}, \quad$ L. J.(2016) L-form bacteria, chronic diseases and the origins of life. Philos. Trans. R. Soc. Lond. B Biol. Sci. 371.

Gilpin, R.W., Young, F.E. and Chatterjee, A.N. (1973) Characterization of a stable L-form of Bacillus subtilis 168. J. Bacteriol. 113, 486-499.

Gomez, J. E., Kaufmann-Malaga, B. B., Wivagg, C. N., Kim, P. B., Silvis, M. R., Renedo, N., Ioerger, T. R., Ahmad, R., Livny, J., Fishbein, S., Sacchettini, J. C., Carr, S. A., Hung, D. T. (2017). Ribosomal mutations promote the evolution of antibiotic resistance in a multidrug environment. eLife, 6 , e20420.

Harms, A., Maisonneuve, E., Gerdes, K., (2016). Mechanisms of bacterial persistence during stress and antibiotic exposure, Science, 354(6318).
Kawai, Y., Mickiewicz, K. \& Errington, J.(2018) Lysozyme counteracts $\beta$ Lactam antibiotics by promoting the emergence of L-form bacteria. Cell 172,1038-1049.

Klieneberger E. (1935) The natural occurrence of pleuropneumonia-like organisms in apparent symbiosis with Streptobacillus moniliformis andother bacteria. J. Pathol.Bacteriol. 40, 93-105.

Losos, J., Mason, K., Singer, S., Raven, P. \& Johnson, G. (2008) Biology (McGrawHill Higher Education, Boston

Lovering A.L.Safadi S.S.Strynadka N.C. (2012)Structural perspective of peptidoglycan biosynthesis and assembly.Annu. Rev. Biochem.; 81: 451-478.

Mercier, R., Kawai, Y. \& Errington, J.(2014) General principles for the formation and proliferation of a wall-free (Lform) state in bacteria. Elife 3, e04629.

Mercier, R., Kawai, Y. \& Errington, J. (2013)Excess membrane synthesis drives a primitive mode of cell proliferation. Cell 152, 997-1007.

Mickiewicz, K.M., Kawai, Y., Drage, L. et al. (2019)Possible role of L-form switching in recurrent urinary tract infection. Nat Commun 10, 4379.

Onwuamaegbu, M. E., Belcher, R. A. \& Soare, C.(2005) Cell wall-deficient bacteria as a cause of infections: a review of the 
clinical significance. J. Int. Med. Res. 33,1-20 .

Plischke M, Kohl M, Bankir L, Shayganfar S, Handisurya A, et al. (2014) Urine Osmolarity and Risk of Dialysis Initiation in a Chronic Kidney Disease Cohort - a Possible Titration Target? PLOS ONE 9(3): e93226.

Prescott, L. M., J. P. Harley, and D. A. Klein. 2005. Microbiology, 6th ed. McGraw-Hill Publishing,NewYork.

Purcell A (18 March 2016). "Bacteria". Basic Biology.

Reygaert WC. (2018)An overview of the antimicrobial resistance mechanisms of bacteria. AIMS Microbiol. 4(3):482501.

Razin S. Mycoplasmas. (1996). Medical Microbiology. 4th edition. Galveston (TX): University of Texas Medical Branch at Galveston.

Rojas ER, Billings G, Odermatt PD, Auer GK, Zhu L, Miguel A, Chang F, Weibel DB, Theriot JA, Huang KC.(2018) The outer membrane is an essential loadbearing element in Gram-negative bacteria. Nature.559(7715):617-621.

Scheffers, D. J., and Pinho, M. G. (2005). Bacterial cell wall synthesis: new insights from localization studies. Microbiology and molecular biology reviews : MMBR, 69(4), 585607.
Tortora, G. J., B. R. Funke, and C. L. Case. 2007. Microbiology: an introduction, 9th ed. Pearson Education, Inc., San Francisco, Calif.

Typas, A., Banzhaf, M., Gross, C. A. \& Vollmer, W. (2011)From the regulation of peptidoglycan synthesis to bacterial growth and morphology. Nat. Rev. Microbiol. 10, 123-136.

World Health Organization. Global action plan on antimicrobial resistance. 2015. 